PROCEEDINGS OF THE

AMERICAN MATHEMATICAL SOCIETY

Volume 134, Number 4, Pages 1117-1124

S 0002-9939(05)08113-X

Article electronically published on August 29, 2005

\title{
A MULTIPLICITY THEOREM FOR THE NEUMANN PROBLEM
}

\author{
BIAGIO RICCERI
}

(Communicated by David S. Tartakoff)

\begin{abstract}
Here is a particular case of the main result of this paper: Let $\Omega \subset$ $\mathbb{R}^{n}$ be a bounded domain, with a boundary of class $C^{2}$, and let $f, g: \mathbb{R} \rightarrow \mathbb{R}$ be two continuous functions, $\alpha \in L^{\infty}(\Omega)$, with ess $\inf _{\Omega} \alpha>0, \beta \in L^{p}(\Omega)$, with $p>n$. If
\end{abstract}

$$
\lim _{|\xi| \rightarrow+\infty} \frac{f(\xi)}{\xi}=0
$$

and if the set of all global minima of the function $\xi \rightarrow \frac{\xi^{2}}{2}-\int_{0}^{\xi} f(t) d t$ has at least $k \geq 2$ connected components, then, for each $\lambda>0$ small enough, the Neumann problem

$$
\begin{cases}-\Delta u=\alpha(x)(f(u)-u)+\lambda \beta(x) g(u) & \text { in } \Omega, \\ \frac{\partial u}{\partial \nu}=0 & \text { on } \partial \Omega\end{cases}
$$

admits at least $k+1$ strong solutions in $W^{2, p}(\Omega)$.

\section{INTRODUCTION}

Here and in the sequel, $\Omega \subset \mathbb{R}^{n}(n \geq 2)$ is an open, bounded and connected set, with boundary of class $C^{2}$.

Let $\varphi, g: \Omega \times \mathbb{R} \rightarrow \mathbb{R}$ be two Carathéodory functions. Since [1], in connection with the Dirichlet problem

$$
\begin{cases}-\Delta u=\varphi(x, u)+\lambda g(x, u) & \text { in } \Omega, \\ u=0 & \text { on } \partial \Omega\end{cases}
$$

a rather classical issue is to find suitable conditions on $\varphi, g$ under which the following is true: for each $k \in \mathbb{N}$ there is $\delta>0$ such that the problem has at least $k$ solutions for all $\lambda \in(0, \delta)$. Among the most recent contributions, we refer to [3] and [4. Note that a common feature of the quoted papers is the assumption that, for each $x \in \Omega$, the function $\varphi(x, \cdot)$ be odd.

As far as we know, it seems that the analogous question about the Neumann problem

$$
\begin{cases}-\Delta u=\varphi(x, u)+\lambda g(x, u) & \text { in } \Omega \\ \frac{\partial u}{\partial \nu}=0 & \text { on } \partial \Omega\end{cases}
$$

Received by the editors June 10, 2004 and, in revised form, November 2, 2004.

2000 Mathematics Subject Classification. Primary 35J20, 35J65.

Key words and phrases. Neumann problem, multiplicity of solutions, global minima, connected components.

(C)2005 American Mathematical Society Reverts to public domain 28 years from publication 
( $\nu$ being the outer unit normal to $\partial \Omega$ ) has not been studied.

The aim of the present paper is to offer a contribution along this direction.

We impose no growth condition on $g$ (as in [3], 4]) and no symmetry condition on $\varphi$. This, in particular, allows us to get nonnegative solutions when $\varphi(x, 0)=$ $g(x, 0)=0$. Our key assumption on $\varphi$ is of a completely different nature. Indeed, we require that $\varphi(x, \xi)=\alpha(x) \psi(\xi)$, where the set of all global minima of the function $\xi \rightarrow \int_{0}^{\xi} \psi(t)$ has infinitely many connected components. Furthermore, our approach (based on a general multiplicity result of [5]) allows us to get information about the location of the solutions.

For instance, here is a consequence of our main result.

Proposition 1. Let $\psi:\left[0,+\infty\left[\rightarrow \mathbb{R}\right.\right.$ be a $C^{1}$ periodic function. Set $a=\inf _{[0,+\infty[} \psi$. Assume $\psi(0)>a$.

Then, for every $k \in \mathbb{N}$, for every $\gamma \in] 0,1\left[\right.$, for every $\alpha \in L^{\infty}(\Omega)$, with $\operatorname{ess~}_{\inf _{\Omega}} \alpha>$ 0 , for every $\rho>0$ and for every Carathéodory function $g: \Omega \times[0,+\infty[\rightarrow \mathbb{R}$, with $g(x, 0)=0$, such that $\sup _{|\xi| \leq s}|g(\cdot, \xi)| \in L^{p}(\Omega)$ for some $p>n$ and for all $s>0$, there exists $\lambda^{*}>0$ such that, for each $\left.\lambda \in\right] 0, \lambda^{*}[$, the Neumann problem

$$
\begin{cases}-\Delta u=\frac{\alpha(x) u}{2(\psi(0)-a)}\left(\frac{1}{u^{\gamma}} \psi^{\prime}\left(\frac{1}{u^{\gamma}}\right)-2\left(\psi\left(\frac{1}{u^{\gamma}}\right)-a\right)\right)+\lambda g(x, u) & \text { in } \Omega, \\ \frac{\partial u}{\partial \nu}=0 & \text { on } \partial \Omega\end{cases}
$$

admits at least $k$ nonzero and nonnegative strong solutions in $W^{2, p}(\Omega)$ which belong to the set

$$
\left\{u \in W^{2, p}(\Omega): \frac{1}{2} \int_{\Omega}|\nabla u(x)|^{2} d x+\int_{\Omega} \frac{\alpha(x)|u(x)|^{2}}{2(\psi(0)-a)}\left(\psi\left(\frac{1}{|u(x)|^{\gamma}}\right)-a\right) d x<\rho\right\} .
$$

\section{RESULTS}

Let $h: \Omega \times \mathbb{R} \rightarrow \mathbb{R}$ be a Carathéodory function. As usual, a weak solution of the problem

$$
\begin{cases}-\Delta u=h(x, u) & \text { in } \Omega \\ \frac{\partial u}{\partial \nu}=0 & \text { on } \partial \Omega\end{cases}
$$

is any $u \in W^{1,2}(\Omega)$ such that $h(\cdot, u(\cdot)) \in L^{2}(\Omega)$ and

$$
\int_{\Omega} \nabla u(x) \nabla v(x) d x-\int_{\Omega} h(x, u(x)) v(x) d x=0
$$

for all $v \in W^{1,2}(\Omega)$. A strong solution of the same problem is any $u \in W^{2,2}(\Omega) \cap$ $C^{1}(\bar{\Omega})$ which satisfies the equation almost everywhere in $\Omega$ and the boundary condition pointwise.

Remark 1. Let $h: \Omega \times \mathbb{R} \rightarrow \mathbb{R}$ be a Carathéodory function which is zero over $\Omega \times]-\infty, 0\left[\right.$ and let $\alpha \in L^{\infty}(\Omega)$, with $\operatorname{ess} \inf _{\Omega} \alpha>0$.

If $u \in C^{0}(\bar{\Omega})$ is a weak solution of the problem

$$
\begin{cases}-\Delta u+\alpha(x) u=h(x, u) & \text { in } \Omega \\ \frac{\partial u}{\partial \nu}=0 & \text { on } \partial \Omega\end{cases}
$$

then $u(x) \geq 0$ for all $x \in \Omega$. 
Indeed, arguing by contradiction, suppose that the set $A=\{x \in \Omega: u(x)<0\}$ is nonempty. By continuity, $A$ is also open. Let $v \in C_{0}^{\infty}(A)$. Since $h(x, u(x))=0$ for all $x \in A$, we clearly have

$$
\int_{A} \nabla u(x) \nabla v(x) d x+\int_{A} \alpha(x) u(x) v(x) d x=0 .
$$

By density, this equality holds for any $v \in W_{0}^{1,2}(A)$. On the other hand, clearly $u(x)=0$ for all $x \in \partial A$, and so $u \in W_{0}^{1,2}(A)$. Hence, in particular, we have

$$
\int_{A}|\nabla u(x)|^{2} d x+\int_{A} \alpha(x)|u(x)|^{2} d x=0
$$

from which, since ess $\inf _{A} \alpha>0$, it follows that $u(x)=0$ for all $x \in A$, a contradiction.

Before stating our main result, we recall the statement of the multiplicity theorem of [5] mentioned in the Introduction:

Theorem A (5, Theorem 8). Let $X$ be a reflexive and separable real Banach space, and let $\Phi, \Psi: X \rightarrow \mathbb{R}$ be two sequentially weakly lower semicontinuous and continuously Gâteaux differentiable functionals, with $\Psi$ also coercive. Assume that the functional $\Psi+\lambda \Phi$ satisfies the Palais-Smale condition for every $\lambda>0$ small enough and that the set of all global minima of $\Psi$ has at least $k$ connected components in the weak topology, with $k \geq 2$.

Then, for each $\rho>\inf _{X} \Psi$, there exists $\lambda^{*}>0$ such that, for every $\left.\lambda \in\right] 0, \lambda^{*}[$, the functional $\Psi+\lambda \Phi$ has at least $k+1$ critical points, $k$ of which are lying in $\Psi^{-1}(]-\infty, \rho[)$.

Our main result reads as follows.

Theorem 1. Let $f: \mathbb{R} \rightarrow \mathbb{R}$ be a continuous function such that

$$
\lim _{|\xi| \rightarrow+\infty} \frac{f(\xi)}{\xi}=0
$$

Assume that the set of all global minima of the function $\xi \rightarrow \frac{\xi^{2}}{2}-\int_{0}^{\xi} f(t) d t$ has at least $k$ connected components, with $k \geq 2$.

Then, for every $\alpha \in L^{\infty}(\Omega)$, with $\operatorname{ess} \inf _{\Omega} \alpha>0$, for every number $\rho$ satisfying

$$
\rho>\|\alpha\|_{L^{1}(\Omega)} \inf _{\xi \in \mathbb{R}}\left(\frac{\xi^{2}}{2}-\int_{0}^{\xi} f(t) d t\right)
$$

and for every Carathéodory function $g: \Omega \times \mathbb{R} \rightarrow \mathbb{R}$ such that $\sup _{|\xi| \leq s}|g(\cdot, \xi)| \in$ $L^{p}(\Omega)$ for some $p>n$ and for all $s>0$, there exists $\lambda^{*}>0$ such that, for each $\lambda \in] 0, \lambda^{*}[$, the Neumann problem

$$
\begin{cases}-\Delta u=\alpha(x)(f(u)-u)+\lambda g(x, u) & \text { in } \Omega \\ \frac{\partial u}{\partial \nu}=0 & \text { on } \partial \Omega\end{cases}
$$

admits at least $k+1$ strong solutions in $W^{2, p}(\Omega), k$ of which belong to the set

$$
\left\{u \in W^{2, p}(\Omega): \frac{1}{2} \int_{\Omega}|\nabla u(x)|^{2} d x+\int_{\Omega} \alpha(x)\left(\frac{|u(x)|^{2}}{2}-\int_{0}^{u(x)} f(t) d t\right) d x<\rho\right\} .
$$


Proof. First, note that, by [7, since $p>n$, there exists $c>0$ such that, for every $\beta \in L^{p}(\Omega)$, the weak solution $u$ of the linear problem

$$
\begin{cases}-\Delta u+\alpha(x) u=\beta(x) & \text { in } \Omega, \\ \frac{\partial u}{\partial \nu}=0 & \text { on } \partial \Omega\end{cases}
$$

satisfies the inequality

$$
\sup _{\Omega}|u| \leq c\|\beta\|_{L^{p}(\Omega)} .
$$

Moreover, this $u$ lies in $W^{2, p}(\Omega)$ (and hence in $C^{1}(\bar{\Omega})$ ) and satisfies the equation a.e. in $\Omega([2,6])$. So, $u$ is a strong solution of the problem. From (1), since $f$ is continuous, it easily follows that

$$
\lim _{r \rightarrow+\infty} \frac{\sup _{|\xi| \leq r}|f(\xi)|}{r}=0 .
$$

Clearly, we also have

$$
\lim _{|\xi| \rightarrow+\infty}\left(\frac{\xi^{2}}{2}-\int_{0}^{\xi} f(t) d t\right)=+\infty .
$$

Now, fix $r>0$ in such a way that

$$
\frac{\sup _{|\xi| \leq r}|f(\xi)|}{r}<\min \left\{1, \frac{1}{c\|\alpha\|_{L^{\infty}(\Omega)}(\operatorname{meas}(\Omega))^{\frac{1}{p}}}\right\}
$$

and that

$$
\left\{s \in \mathbb{R}: \frac{s^{2}}{2}-\int_{0}^{s} f(t) d t=\inf _{\xi \in \mathbb{R}}\left(\frac{\xi^{2}}{2}-\int_{0}^{\xi} f(t) d t\right)\right\} \subset[-r, r] .
$$

Next, put

$$
f_{0}(\xi)= \begin{cases}f(r) & \text { if } \xi>r \\ f(\xi) & \text { if }|\xi| \leq r \\ f(-r) & \text { if } \xi<-r\end{cases}
$$

and

$$
g_{0}(x, \xi)= \begin{cases}g(x, r) & \text { if } \xi>r, x \in \Omega \\ g(x, \xi) & \text { if }|\xi| \leq r, x \in \Omega \\ g(x,-r) & \text { if } \xi<-r, x \in \Omega .\end{cases}
$$

Furthermore, for each $u \in W^{1, p}(\Omega)$, put

$$
\Psi(u)=\frac{1}{2} \int_{\Omega}|\nabla u(x)|^{2} d x+\int_{\Omega} \alpha(x)\left(\frac{|u(x)|^{2}}{2}-\int_{0}^{u(x)} f_{0}(t) d t\right) d x
$$

and

$$
\Phi(u)=-\int_{\Omega}\left(\int_{0}^{u(x)} g_{0}(x, t) d t\right) d x .
$$

By classical results, the functionals $\Psi$ and $\Phi$ are sequentially weakly lower semicontinuous and continuously Gâteaux differentiable in $W^{1,2}(\Omega)$ (equipped with the usual norm $\left.\|u\|=\left(\int_{\Omega}\left(|\nabla u(x)|^{2}+|u(x)|^{2}\right) d x\right)^{\frac{1}{2}}\right)$, the derivative of $\Phi$ is compact, 
and, for every $\lambda \in \mathbb{R}$, the critical points of $\Psi+\lambda \Phi$ are the weak solutions of the problem

$\left(P_{0, \lambda}\right) \quad \begin{cases}-\Delta u+\alpha(x) u=\alpha(x) f_{0}(u)+\lambda g_{0}(x, u) & \text { in } \Omega, \\ \frac{\partial u}{\partial \nu}=0 & \text { on } \partial \Omega .\end{cases}$

For each $u \in W^{1,2}(\Omega)$ and each $\lambda \geq 0$, we clearly have

$$
\begin{aligned}
\Psi(u)+\lambda \Phi(u) \geq \frac{\min \left\{1, \operatorname{ess}_{\inf } \alpha\right\}}{2}\|u\|^{2} & -\|\alpha\|_{L^{\infty}(\Omega)} \sup _{[-r, r]}|f| \int_{\Omega}|u(x)| d x \\
& -\lambda \int_{\Omega} \sup _{|\xi| \leq r}|g(x, \xi) \| u(x)| d x \\
\geq \frac{\min \left\{1, \operatorname{ess} \inf _{\Omega} \alpha\right\}}{2}\|u\|^{2} & \\
& -\left(\|\alpha\|_{L^{\infty}(\Omega)} \sup _{[-r, r]}|f|(\operatorname{meas}(\Omega))^{\frac{1}{2}}\right. \\
& \left.+\lambda\left(\int_{\Omega|\xi| \leq r} \sup _{|\xi|}|g(x, \xi)|^{2} d x\right)^{\frac{1}{2}}\right)\|u\|
\end{aligned}
$$

from which it follows that

$$
\lim _{\|u\| \rightarrow+\infty}(\Psi(u)+\lambda \Phi(u))=+\infty .
$$

This implies, in particular, that the functional $\Psi+\lambda \Phi$ satisfies the Palais-Smale condition ([8], Example 38.25). Now, observe that for $\xi<-r$ one has

$$
\frac{\xi^{2}}{2}-\frac{r^{2}}{2}-f(-r)(\xi+r)>(\xi+r)(-r-f(-r))>0
$$

and so

$$
\frac{\xi^{2}}{2}-\int_{0}^{\xi} f_{0}(t) d t=\frac{\xi^{2}}{2}-\frac{r^{2}}{2}-f(-r)(\xi+r)+\frac{r^{2}}{2}-\int_{0}^{-r} f(t) d t>\frac{r^{2}}{2}-\int_{0}^{-r} f(t) d t .
$$

Analogously, for $\xi>r$, we get

$$
\frac{\xi^{2}}{2}-\frac{r^{2}}{2}-f(r)(\xi-r)>(\xi-r)(r-f(r))>0
$$

and so

$$
\frac{\xi^{2}}{2}-\int_{0}^{\xi} f_{0}(t) d t=\frac{\xi^{2}}{2}-\frac{r^{2}}{2}-f(r)(\xi-r)+\frac{r^{2}}{2}-\int_{0}^{r} f(t) d t>\frac{r^{2}}{2}-\int_{0}^{r} f(t) d t .
$$

Hence, if $|\xi|>r$, we have

$$
\frac{\xi^{2}}{2}-\int_{0}^{\xi} f_{0}(t) d t>\inf _{|\sigma| \leq r}\left(\frac{\sigma^{2}}{2}-\int_{0}^{\sigma} f(t) d t\right) .
$$

From this, thanks to (4), we then infer that

$$
\inf _{\xi \in \mathbb{R}}\left(\frac{\xi^{2}}{2}-\int_{0}^{\xi} f_{0}(t) d t\right)=\inf _{\xi \in \mathbb{R}}\left(\frac{\xi^{2}}{2}-\int_{0}^{\xi} f(t) d t\right)
$$


and that

$$
\begin{aligned}
& \left\{s \in \mathbb{R}: \frac{s^{2}}{2}-\int_{0}^{s} f_{0}(t) d t=\inf _{\xi \in \mathbb{R}}\left(\frac{\xi^{2}}{2}-\int_{0}^{\xi} f_{0}(t) d t\right)\right\} \\
& =\left\{s \in \mathbb{R}: \frac{s^{2}}{2}-\int_{0}^{s} f(t) d t=\inf _{\xi \in \mathbb{R}}\left(\frac{\xi^{2}}{2}-\int_{0}^{\xi} f(t) d t\right)\right\} .
\end{aligned}
$$

Hence, by assumption, the set $\left\{s \in \mathbb{R}: \frac{s^{2}}{2}-\int_{0}^{s} f_{0}(t) d t=\inf _{\xi \in \mathbb{R}}\left(\frac{\xi^{2}}{2}-\int_{0}^{\xi} f_{0}(t) d t\right)\right\}$ (denote it by $M$ ) has at least $k$ connected components. For each $u \in W^{1,2}(\Omega)$, we clearly have

$$
\Psi(u) \geq \inf _{s \in \mathbb{R}}\left(\frac{s^{2}}{2}-\int_{0}^{s} f(t) d t\right)\|\alpha\|_{L^{1}(\Omega)}
$$

and that equality holds if $u$ is almost everywhere equal to a constant $b$ such that $\frac{b^{2}}{2}-\int_{0}^{b} f(t) d t=\inf _{s \in \mathbb{R}}\left(\frac{s^{2}}{2}-\int_{0}^{s} f(t) d t\right)$. Consequently

$$
\inf _{u \in W^{1,2}(\Omega)} \Psi(u)=\inf _{s \in \mathbb{R}}\left(\frac{s^{2}}{2}-\int_{0}^{s} f(t) d t\right)\|\alpha\|_{L^{1}(\Omega)} .
$$

On the other hand, if $u \in W^{1,2}(\Omega)$ is not almost everywhere equal to a constant, then $|\nabla u|>0$ in some set of positive measure (recall that $\Omega$ is connected). From this, it clearly follows that

$$
\left\{u \in W^{1,2}(\Omega): \Psi(u)=\inf _{s \in \mathbb{R}}\left(\frac{s^{2}}{2}-\int_{0}^{s} f(t) d t\right)\|\alpha\|_{L^{1}(\Omega)}\right\}=\gamma(M)
$$

where $\gamma$ denotes the mapping that to each $r \in \mathbb{R}$ associates the equivalence class of functions almost everywhere equal in $\Omega$ to $r$. Of course, the mapping $\gamma$ is a homeomorphism between $\mathbb{R}$ and $\gamma(\mathbb{R})$, this latter set being considered with the relativization of the weak topology on $W^{1,2}(\Omega)$. Hence, the set of all global minima of the functional $\Psi$ has at least $k$ connected components in the weak topology. Summarizing: taking $X=W^{1,2}(\Omega)$, the functionals $\Psi$ and $\Phi$ satisfy all the assumptions of Theorem A. Consequently, there exists $\lambda^{*}>0$ such that, for each $\lambda \in] 0, \lambda^{*}\left[\right.$, problem $\left(P_{0, \lambda}\right)$ has at least $k+1$ weak solutions, $k$ of which belong to the set $\Psi^{-1}(]-\infty, \rho[)$. Now, observe that if $u$ is any weak solution of problem $\left(P_{0, \lambda}\right)$, then, by $(2)$, one has

$$
\sup _{\Omega}|u| \leq c\left(\|\alpha\|_{L^{\infty}(\Omega)} \max _{[-r, r]}|f|(\operatorname{meas}(\Omega))^{\frac{1}{p}}+\lambda\left(\int_{\Omega} \sup _{|\xi| \leq r}|g(x, \xi)|^{p} d x\right)^{\frac{1}{p}}\right) .
$$

On the other hand, by (3), one has

$$
c\|\alpha\|_{L^{\infty}(\Omega)} \max _{[-r, r]}|f|(\operatorname{meas}(\Omega))^{\frac{1}{p}}<r .
$$

Consequently, if $\lambda$ satisfies

$$
\lambda<\frac{r-c\|\alpha\|_{L^{\infty}(\Omega)} \max _{[-r, r]}|f|(\operatorname{meas}(\Omega))^{\frac{1}{p}}}{c\left(\int_{\Omega} \sup _{|\xi| \leq r}|g(x, \xi)|^{p} d x\right)^{\frac{1}{p}}}
$$


then, from (5), we get

$$
\sup _{\Omega}|u|<r,
$$

and so $u$ is a weak solution of problem $\left(P_{\lambda}\right)$. From this, the conclusion follows.

Remark 2. The key assumption of Theorem 1 is that the set of all global minima of the function $\xi \rightarrow \frac{\xi^{2}}{2}-\int_{0}^{\xi} f(t) d t$ has at least $k$ connected components. Knowing simply that this set is infinite is not useful in order to determine the multiplicity of the solutions of the considered Neumann problem. In this connection, consider the function $f: \mathbb{R} \rightarrow \mathbb{R}$ defined by

$$
f(\xi)= \begin{cases}0 & \text { if } \xi<0 \\ \xi & \text { if } \xi \in[0,1] \\ 1 & \text { if } \xi>1\end{cases}
$$

Since

$$
\frac{\xi^{2}}{2}-\int_{0}^{\xi} f(t) d t= \begin{cases}\frac{\xi^{2}}{2} & \text { if } \xi<0, \\ 0 & \text { if } \xi \in[0,1], \\ \frac{(\xi-1)^{2}}{2} & \text { if } \xi>1,\end{cases}
$$

the set of all global minima of the function $\xi \rightarrow \frac{\xi^{2}}{2}-\int_{0}^{\xi} f(t) d t$ is $[0,1]$. Nevertheless, for each $\lambda>0$, the functional

$$
\begin{aligned}
u \rightarrow \frac{1}{2} \int_{\Omega}|\nabla u(x)|^{2}+\int_{\Omega}\left(\frac{|u(x)|^{2}}{2}-\int_{0}^{u(x)} f(t) d t\right) d x+\frac{\lambda}{2} \int_{\Omega}|u(x)|^{2} d x, \\
u \in W^{1,2}(\Omega),
\end{aligned}
$$

is strictly convex, and so it has a unique critical point (its global minimum). That is, the Neumann problem

$$
\begin{cases}-\Delta u=f(u)-u+\lambda u & \text { in } \Omega, \\ \frac{\partial u}{\partial \nu}=0 & \text { on } \partial \Omega\end{cases}
$$

has a unique solution.

It is worth noticing the following consequence of Theorem 1.

Theorem 2. Let $f:[0,+\infty[\rightarrow \mathbb{R}$ be a continuous function satisfying $f(0)=0$ and

$$
\lim _{\xi \rightarrow+\infty} \frac{f(\xi)}{\xi}=0 .
$$

Assume that the set of all global minima of the function $\xi \rightarrow \frac{\xi^{2}}{2}-\int_{0}^{\xi} f(t) d t$ has at least $k$ connected components, with $k \geq 2$.

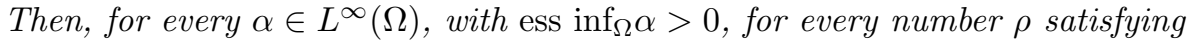

$$
\rho>\|\alpha\|_{L^{1}(\Omega)} \inf _{\xi \in[0,+\infty[}\left(\frac{\xi^{2}}{2}-\int_{0}^{\xi} f(t) d t\right)
$$

and for every Carathéodory function $g: \Omega \times[0,+\infty[\rightarrow \mathbb{R}$, with $g(x, 0)=0$, such that $\sup _{|\xi| \leq s}|g(\cdot, \xi)| \in L^{p}(\Omega)$ for some $p>n$ and for all $s>0$, there exists $\lambda^{*}>0$ 
such that, for each $\lambda \in] 0, \lambda^{*}[$, the Neumann problem

$$
\begin{cases}-\Delta u=\alpha(x)(f(u)-u)+\lambda g(x, u) & \text { in } \Omega \\ \frac{\partial u}{\partial \nu}=0 & \text { on } \partial \Omega\end{cases}
$$

admits at least $k$ nonzero and nonnegative solutions in $W^{2, p}(\Omega), k-1$ of which belong to the set

$$
\left\{u \in W^{2, p}(\Omega): \frac{1}{2} \int_{\Omega}|\nabla u(x)|^{2} d x+\int_{\Omega} \alpha(x)\left(\frac{|u(x)|^{2}}{2}-\int_{0}^{u(x)} f(t) d t\right) d x<\rho\right\} .
$$

Proof. Denote by $f_{1}$ and $g_{1}$ the extensions of $f$ and $g$ to $\mathbb{R}$ and $\Omega \times \mathbb{R}$ which are zero over ] $-\infty, 0[$ and $\Omega \times]-\infty, 0$ [, respectively. Clearly, $f_{1}$ satisfies the assumptions of Theorem 1 , with the same $k$. Now, the conclusion follows directly from Theorem 1 via Remark 1.

Proof of Proposition 1. Define the function $f:[0,+\infty[\rightarrow \mathbb{R}$ by

$$
f(\xi)= \begin{cases}\xi+\frac{\xi}{2(\psi(0)-a)}\left(\frac{1}{\xi^{\gamma}} \psi^{\prime}\left(\frac{1}{\xi^{\gamma}}\right)-2\left(\psi\left(\frac{1}{\xi^{\gamma}}\right)-a\right)\right) & \text { if } \xi>0, \\ 0 & \text { if } \xi=0 .\end{cases}
$$

As $\gamma \in] 0,1[, f$ is continuous and (1) holds. Since

$$
\frac{\xi^{2}}{2}-\int_{0}^{\xi} f(t) d t=\frac{\xi^{2}}{2(\psi(0)-a)}\left(\psi\left(\frac{1}{\xi^{\gamma}}\right)-a\right)
$$

for all $\xi>0$, due to the periodicity of $\psi$, the set of all global minima of the function $\xi \rightarrow \frac{\xi^{2}}{2}-\int_{0}^{\xi} f(t) d t$ (that is, the set $\left\{\xi>0: \psi\left(\frac{1}{\xi^{\gamma}}\right)=a\right\}$ ) has infinitely many connected components. Now, the conclusion follows directly from Theorem 2 , which is possible to apply with any integer $k \geq 2$.

\section{REFERENCES}

1. A. Ambrosetti, A perturbation theorem for superlinear boundary value problems, M. R. C. Tech. Summ. Rep. (1974).

2. D. Gilbarg and N. S. Trudinger, Elliptic partial differential equations of second order, Springer-Verlag, 2001. MR.1814364 (2001k:35004)

3. S. J. Li and Z. L. Liu, Perturbations from symmetric elliptic boundary value problems, J. Differential Equations 185 (2002), 271-280. MR1935639 (2003h:35081)

4. Z. L. Liu and J. B. Su, Solutions of some nonlinear elliptic problems with perturbation terms of arbitrary growth, Discrete Contin. Dyn. Syst. 10 (2004), 617-634. MR2018870(2005b:35096)

5. B. Ricceri, Sublevel sets and global minima of coercive functionals and local minima of their perturbations, J. Nonlinear Convex Anal. 5 (2004), 157-168. MR2083908

6. C. G. Simader, Higher regularity of weak $L_{q}$-solutions of the Neumann problem for the Laplacian, Bayreuth. Math. Schr., to appear.

7. G. Stampacchia, Contributi alla regolarizzazione delle soluzioni dei problemi al contorno per equazioni del secondo ordine ellittiche, Ann. Scuola Norm. Sup. Pisa (3) 12 (1958), 223-245. MR0125313 (23:A2616)

8. E. Zeidler, Nonlinear functional analysis and its applications, vol. III, Springer-Verlag, 1985. MR0768749 (90b:49005)

Department of Mathematics, University of Catania, Viale A. Doria 6, 95125 Catania, ITALY

E-mail address: ricceri@dmi.unict.it 